\title{
Intravitreal ranibizumab, photodynamic therapy, and vitreous surgery for the treatment of juxtapapillary retinal capillary hemangioma
}

\author{
Angie H. C. Fong • Kenneth K. W. Li • David Wong
}

Received: 31 August 2009 /Revised: 5 July 2010 /Accepted: 7 July 2010 / Published online: 31 July 2010

(C) The Author(s) 2010. This article is published with open access at Springerlink.com

\section{Dear Editor,}

Retinal capillary hemangiomas ( $\mathrm{RCH})$ are capillary angiomatous hamartomas of the retina and optic nerve head. The median age of diagnosing the disease is 31 years old [1]. Although most RCH is isolated, $10-30 \%$ in the $31-40$ age group are associated with Von Hippel Lindau disease (VHL). Up to $15 \%$ of RCHs are juxtapapillary, where most are located temporally [2]. They are often associated with epiretinal membrane (ERM) formation and serous and tractional retinal detachment of the macula, which leads to a poor prognosis [1].

We report a typical case of isolated juxtapapillary $\mathrm{RCH}$ that underwent successful combined therapy with intravitreal ranibizumab injection and photodynamic therapy (PDT) 1 week before PPV and ERM peeling surgery.

A 32-year-old woman presented with reduced right eye vision and metamorphopsia for 3 months. At presentation, her best-corrected visual acuity was 20/50 and 20/25 for the

Financial and proprietary interests: None

A. H. C. Fong $\cdot$ K. K. W. Li $\cdot$ D. Wong

Department of Ophthalmology, Queen Mary Hospital,

Pokfulam,

Hong Kong SAR, China

K. K. W. Li $(\bowtie) \cdot$ D. Wong

Department of Ophthalmology, United Christian Hospital,

Kowloon,

Hong Kong SAR, China

e-mail: kennethli@rcsed.ac.uk

K. K. W. Li $\cdot$ D. Wong

Eye Institute, Li Ka Shing Faculty of Medicine,

University of Hong Kong,

Hong Kong SAR, China right and left eyes, respectively. Right eye fundus examination revealed a reddish mass measuring $2.0 \times 1.5 \mathrm{~mm}$ in the inferotemporal juxtapapillary region associated with macular subretinal fluid and ERM (Fig. 1a).

Fundus fluorescein angiogram (FFA) showed early filling of the lesion with late leakage into the subretinal space. Anomalous vascular channels were seen sprouting from the lesion (Fig. 1b). Optical coherence tomography (OCT) confirmed serous detachment of the macula with vitreomacular traction (Fig. 1c). Central foveal thickness was increased to $570 \mu \mathrm{m}$ (normal values $=<315 \mu \mathrm{m}$ ) [3].

A diagnosis of juxtapapillary retinal capillary hemangioma was made. Systemic workup for Von Hippel Lindau (VHL) disease did not reveal any other tumors. Her visual acuity continued to deteriorate to $20 / 100$ in the subsequent 2 months. She was treated once with PDT using standard fluence at that time, with reduced diameter laser spot centering on the lesion avoiding the optic disc. There was no clinical response and her vision continued to deteriorate to 20/200 3 months after the initial PDT. Combined therapy, using standard fluence PDT and intravitreal injection of ranibizumab were performed 2 months later (7 months since presentation). Reduction of lesion size $(1.5 \times 1.5 \mathrm{~mm})$ and vascularity was noted 1 day after the combined therapy (Fig. 2a). One week later, PPV and ERM peel were carried out and intravitreal triamcinolone injection $(2 \mathrm{mg}$ in $0.05 \mathrm{ml}$ ) was given at the conclusion of surgery.

The patient's visual acuity improved to 20/25 after successful surgery and the lesion remained stable for 12 months, up to the latest follow-up (Fig. 2b). The central foveal thickness remained reduced within normal limits at $324 \mu \mathrm{m}$. FFA showed hyperfluorescence in the late phase (Fig. 2c). A small superotemporal branch retinal vein 

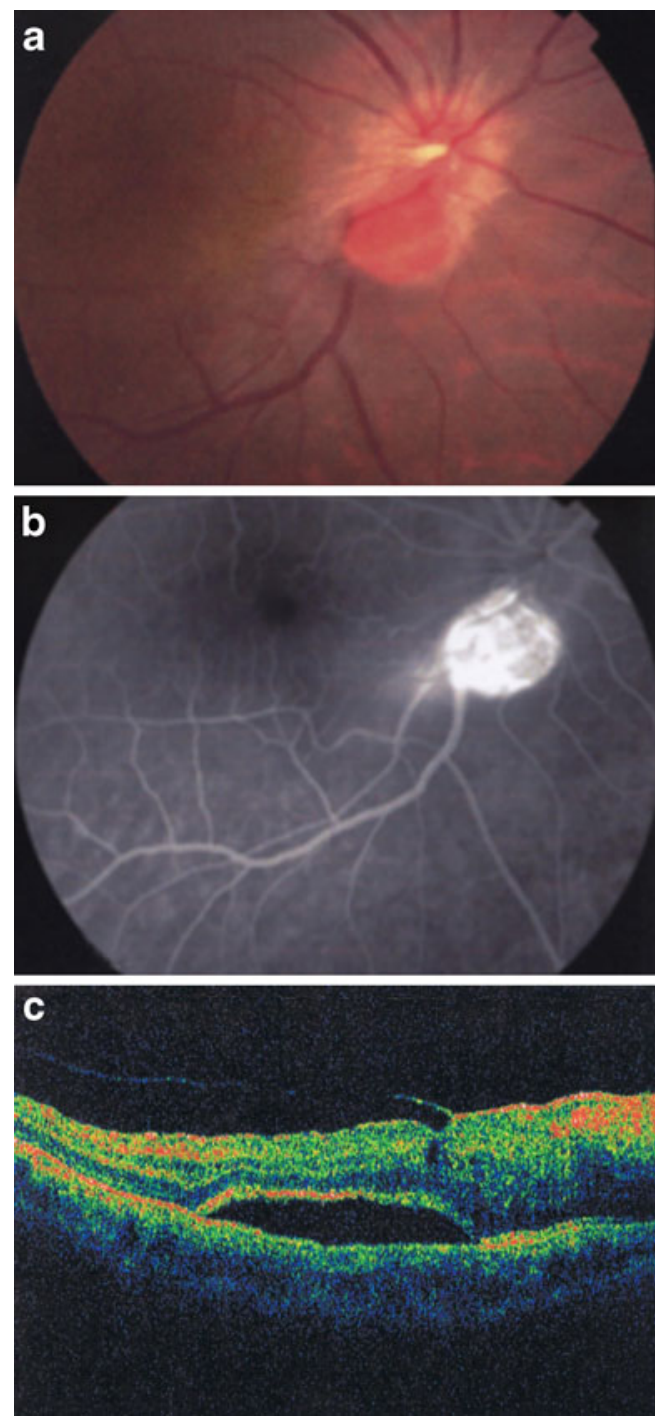

Fig. 1 Imaging results (color fundus photography, FFA and OCT) of the patient at initial presentation. a Fundus photograph of the right eye showing juxtapapillary retinal capillary hemangioma, measured $2.0 \times$ $1.5 \mathrm{~mm}$, located inferotemporal to the optic disc. b Fundus fluorescein angiography demonstrating hyperflourescence of the lesion as well as late leakage into submacular space. c OCT (horizontal 5-mm scan across the fovea) showing macular detachment secondary to vitreomacular traction

occlusion developed secondary to an iatrogenic break resolved spontaneously.

\section{Discussion}

To date, no single modality in the treatment of juxtapapillary RCH has been particularly effective [1]. Laser photocoagulation applied to the neural tissue surrounding the tumor can cause permanent scotomas [2]. Use of cryotherapy is limited due to the posterior location of the lesion.
Anti-vascular endothelial growth factor (anti-VEGF) therapy has been reported to reduce vascular permeability by altering the balance of vaso-active cytokines like nitric
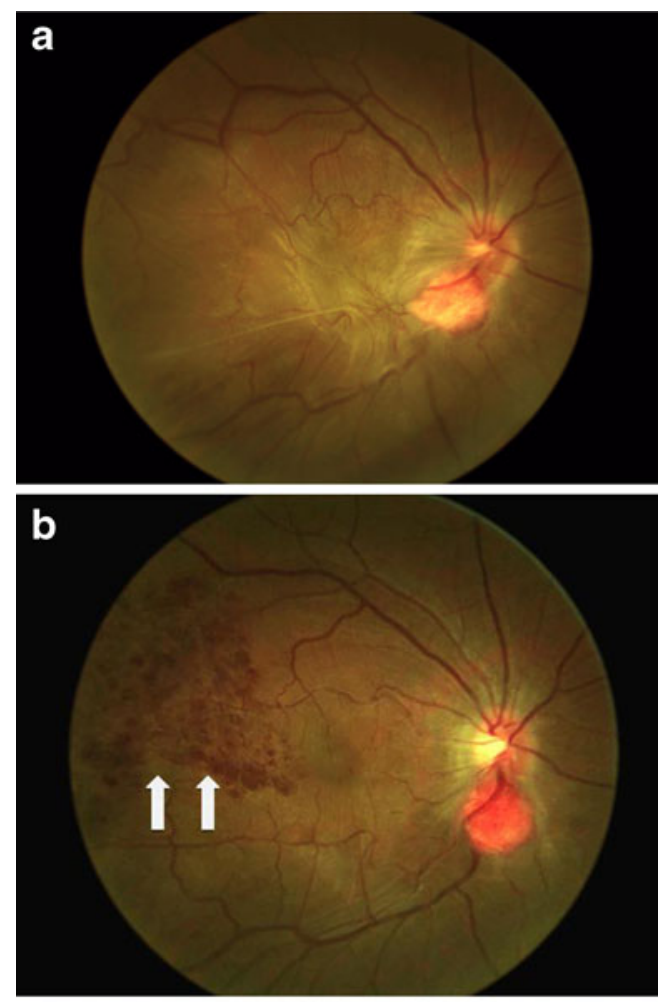

C

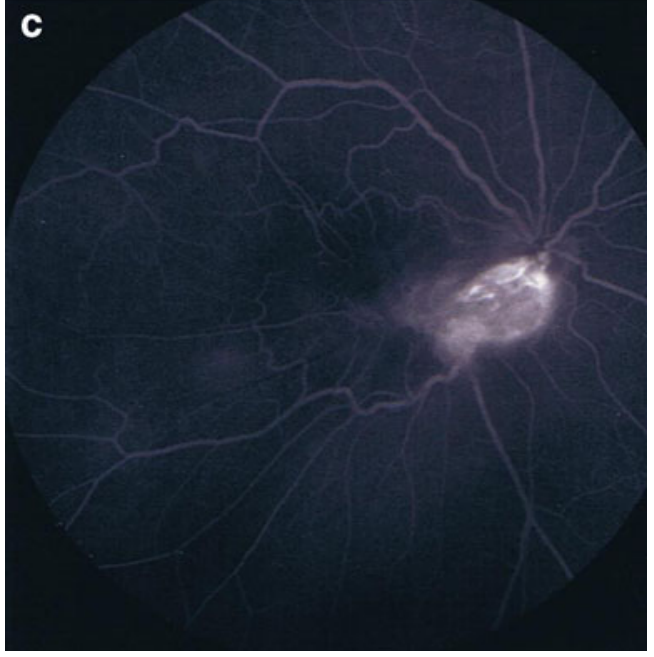

Fig. 2 Imaging results (color fundus photography and FFA) of the patient after combined treatment with injection of ranibizumab, PDT, and intravitreal surgery. a Day 1 after combined intravitreal ranibizumab injection and PDT, showing regression of the tumor as well as marked decrease in vascularity and exudation. b Post-operative (month 2) fundus photograph showing relief of traction on the macula after removal of the epiretinal membrane and reduction in size of the angioma. A peripheral branch retinal vein occlusion (white arrows) resulted from a small iatrogenic break. c Late phase of fundus flourescein angiography (month 12) showing staining of the lesion with minimal leakage 
oxide and endothelin-1 [4] or by directly altering endothelial tight junction proteins [5]. It is postulated that excessive accumulation of hypoxic induced factor (HIF) in the neoplastic stromal cells of RCH leads to the production of other angiogenic factors that are able to maintain and promote the growth of primary hemangiomas [6].

PDT is shown to be effective in causing fibrosis and involution of the primary angioma, but its use on peripapillary area is limited by vaso-occlusive effects $[2,7]$. For larger tumors, verteporfin may only be activated on the surface of the tumor, and the reactive oxygen species may not cause closure of deeper tumor vessels. By combining anti-VEGF with reduced fluence PDT, the outline of the primary angioma can be better delineated and may thus reduce the energy and the treatment area, thereby minimizing the damage to the neurological tissues [8]. Recent reports of combined therapy with anti-VEGF therapy and PDT have shown promising results in these lesions [7].

Anti-VEGF agents, like bevacizumab, have been reported as a useful pre-operative adjunct in the surgical treatment of proliferative diabetic retinopathy, with significant reduction in neovascularization, bleeding, and adherence of fibrovascular complex to the retina [9]. We found that ranibizumab is useful in the present case. Combined treatment with anti-VEGF and PDT 1 week before surgery caused a reduction in macular edema, which reduced the risk of retinal cyst rupture during peeling procedure [10]. Reduced tumor vascularization may have lowered the risk of intraoperative bleeding.

In summary, pre-operative combination therapy with an anti-VEGF agent and PDT, PPV, and ERM followed by intravitreal triamcinolone at the conclusion of surgery led to improvement in visual outcome. The use of triple therapy was recently shown to be effective in the treatment of agerelated macular degeneration and our case may suggest that a multi-faceted approach could be beneficial in cases of juxtapapillary RCH [11].

Open Access This article is distributed under the terms of the Creative Commons Attribution Noncommercial License which permits any noncommercial use, distribution, and reproduction in any medium, provided the original author(s) and source are credited.

\section{References}

1. Singh AD, Nouri M, Shields CL, Shields JA, Perez N (2002) Treatment of retinal capillary hemangioma. Ophthalmology 109 (10):1799-1806

2. Schmidt-Erfurth UM, Kusserow C, Barbazetto IA, Laqua H (2002) Benefits and complications of photodynamic therapy of papillary capillary hemangiomas. Ophthalmology 109(7):1256-1266

3. Grover S, Murthy RK et al (2009) Normative data for macular thickness by high-definition spectral-domain optical coherence tomography (spectralis). Am J Ophthalmol 148(2):266-271

4. Warner TD (1999) Relationships between the endothelin and nitric oxide pathways. Clin Exp Pharmacol Physiol 26(3):247-252

5. Wang W, Dentler WL, Borchardt RT (2001) VEGF increases BMEC monolayer permeability by affecting occludin expression and tight junction assembly. Am J Physiol Heart Circ Physiol 280(1):434-440

6. Chan CC, Collins AB, Chew EY (2007) Molecular pathology of eyes with von Hippel-Lindau (VHL) disease: a review. Retina 27(1):1-7

7. Mennel S, Meyer CH, Callizo J (2009) Combined intravitreal antivascular endothelial growth factor (Avastin) and photodynamic therapy to treat retinal juxtapapillary capillary haemangioma. Acta Ophthalmol Feb 12 [Epub ahead of print]

8. Tzekov R, Lin T, Zhang KM, Jackson B, Oyejide A, Orilla W, Kulkarni AD, Kuppermann BD, Wheeler L, Burke J (2006) Ocular changes after photodynamic therapy. Invest Ophthalmol Vis Sci 47(1):377-385

9. Figueroa MS, Contreras I, Noval S (2009) Anti-angiogenic drugs as an adjunctive therapy in the surgical treatment of diabetic retinopathy. Curr Diabetes Rev 5(1):52-56

10. Steven P, Laqua H, Wong D, Hoerauf H (2006) Secondary paracentral retinal holes following internal limiting membrane removal. Br J Ophthalmol 90(3):293-295

11. Bakri SJ, Couch SM et al (2009) Same-day triple therapy with photodynamic therapy, intravitreal dexamethasone, and bevacizumab in wet age-related macular degeneration. Retina 29(5):573-578 